\title{
Development of a marker panel for genotyping of soybean cultivars by genes controlling the flowering time and response to the photoperiod
}

\author{
Perfil'ev R.N. ${ }^{1,2 *}$, Shcherban A.B. ${ }^{1}$, Salina E.A. ${ }^{1}$ \\ ${ }^{1}$ Institute of Cytology and Genetics, SB RAS, Novosibirsk, Russia \\ ${ }^{2}$ Novosibirsk State University, Novosibirsk, Russia \\ * email: PerfilyevRN@bionet.nsc.ru
}

Soybean is a short-day plant with a very strong reaction to the day length. The duration of daylight hours most strongly affects the interfacial period between seedlings and flowering. This phase of growth and development is critical and determines the future maturity of the variety. A large number of genes and QTLs controls the photoperiod response. For genes with the strongest effect $E 1, E 2, E 3, E 4$ and with the least effect $E 9$, GmFT5a, the molecular structure and natural alleles, which cause variations in the growing season, were determined. All this makes it possible to develop and use allelespecific molecular markers for these genes in order to study the gene pool and marker assisted selection. For these purposes, we have developed an expanded panel of PCR molecular markers for the indicated genes and their most common alleles. The panel consists of original PCR markers for genes E1 and GmFT5a, and known PCR markers for other genes. When developing original PCR markers, the allele in the cultivars was additionally, define by sequencing the site with the allele-determining nucleotide substitution. Using this panel, 10 promising varieties of Russian and foreign selection were genotyped. Varieties from the collection of the Siberian Research Institute of Forage with a group of maturity from MG 0 to MG 000 The obtained genotype data will allow us and other breeders to use this information to select parental pairs when crossing, taking into account the possibility of achieving the required genotype in the offspring. The developed original PCR markers will make it possible to distinguish functionally different alleles in the E1 and GmFT5a genes. In general, the expanded panel of molecular markers will allow highly efficient marker assisted breeding to create varieties with the required maturity group.

Acknowledgements: This work was done within the framework of State Assignment Kurchatov Genomic Center of the Institute of Cytology and Genetics, SB RAS (075-152019-1662). 